\title{
Comparison of MRI Lesion Evolution in Different Central Nervous System Demyelinating Disorders
}

\begin{abstract}
Elia Sechi, MD, Karl N. Krecke, MD, Steven A. Messina, MD, Marina Buciuc, MD, Sean J. Pittock, MD, John J. Chen, MD, PhD, Brian G. Weinshenker, MD, A. Sebastian Lopez-Chiriboga, MD,

Claudia F. Lucchinetti, MD, Nicholas L. Zalewski, MD, Jan Mendelt Tillema, MD, Amy Kunchok, MD, Salvatore Monaco, MD, Padraig P. Morris, MD, James P. Fryer, MS, Adam Nguyen, Tammy Greenwood, MS, Stephanie B. Syc-Mazurek, MD, PhD, B. Mark Keegan, MD, and Eoin P. Flanagan, MD
\end{abstract}

Neurology ${ }^{\circledR}$ 2021;97:e1097-e1109. doi:10.1212/WNL.0000000000012467

\section{Abstract}

\section{Background and Objective}

There are few studies comparing lesion evolution across different CNS demyelinating diseases, yet knowledge of this may be important for diagnosis and understanding differences in disease pathogenesis. We sought to compare MRI T2 lesion evolution in myelin oligodendrocyte glycoprotein immunoglobulin $\mathrm{G}(\mathrm{IgG})$-associated disorder (MOGAD), aquaporin $4 \mathrm{IgG}$-positive neuromyelitis optica spectrum disorder (AQP4-IgG-NMOSD), and multiple sclerosis (MS).

\section{Methods}

In this descriptive study, we retrospectively identified Mayo Clinic patients with MOGAD, AQP4-IgG-NMOSD, or MS and (1) brain or myelitis attack; (2) available attack MRI within 6 weeks; and (3) follow-up MRI beyond 6 months without interval relapses in that region. Two neurologists identified the symptomatic or largest T2 lesion for each patient (index lesion). MRIs were then independently reviewed by 2 neuroradiologists blinded to diagnosis to determine resolution of $\mathrm{T} 2$ lesions by consensus. The index $\mathrm{T} 2$ lesion area was manually outlined acutely and at follow-up to assess variation in size.

\section{Results}

We included 156 patients (MOGAD, 38; AQP4-IgG-NMOSD, 51; MS, 67) with 172 attacks (brain, 81; myelitis, 91). The age (median [range]) differed between MOGAD (25 [2-74]), AQP4-IgG-NMOSD (53 [10-78]), and MS (37 [16-61]) $(p<0.01)$ and female sex predominated in the AQP4-IgG-NMOSD (41/51 [80\%]) and MS (51/67 [76\%]) groups but not among those with MOGAD (17/38 [45\%]). Complete resolution of the index T2 lesion was more frequent in MOGAD (brain, 13/18 [72\%]; spine, 22/28 [79\%]) than AQP4-IgGNMOSD (brain, 3/21 [14\%]; spine, 0/34 [0\%]) and MS (brain, 7/42 [17\%]; spine, 0/29 $[0 \%])(p<0.001)$. Resolution of all T2 lesions occurred most often in MOGAD (brain, 7/18 [39\%]; spine, 22/28 [79\%]) than AQP4-IgG-NMOSD (brain, 2/21 [10\%]; spine, 0/34 [0\%]) and MS (brain, 2/42 $[5 \%]$; spine, $0 / 29[0 \%])(p<0.01)$. There was a larger median (range) reduction in T2 lesion area in $\mathrm{mm}^{2}$ on follow-up axial brain MRI with MOGAD (213 [55-873]) than AQP4-IgG-NMOSD (104 [0.7-597]) $(p=0.02)$ and MS (36 [0-506]) $(p<$ $0.001)$ and the reductions in size on sagittal spine MRI follow-up in MOGAD (262 [0-888]) and AQP4-IgG-NMOSD (309 [0-1885]) were similar $(p=0.4)$ and greater than in MS (23 $[0-152])(p<0.001)$.

\author{
Correspondence \\ Dr. Flanagan \\ flanagan.eoin@mayo.edu
}

MORE ONLINE

\section{(ค) Podcast}

Dr. Stacey Clardy discusses the paper "Comparison of MRI Lesion Evolution in Different Central Nervous System Demyelinating Disorders" with Dr. Eoin Flanagan.

NPub.org/lg0etl 


\section{Glossary}

AQP4 = aquaporin 4; AQP4-IgG-NMOSD = aquaporin 4-immunoglobulin G-positive neuromyelitis optica spectrum disorder; EDSS = Expanded Disability Status Scale; FLAIR = fluid-attenuated inversion recovery; IgG = immunoglobulin G; IVIg = IV immunoglobulin; MOG = myelin oligodendrocyte glycoprotein; MOGAD = myelin oligodendrocyte glycoprotein-immunoglobulin G-associated disorder; $\mathbf{M S}$ = multiple sclerosis; PLEX = plasma exchange.

\section{Discussion}

The MRI T2 lesions in MOGAD resolve completely more often than in AQP4-IgG-NMOSD and MS. This has implications for diagnosis, monitoring disease activity, and clinical trial design, while also providing insight into pathogenesis of CNS demyelinating diseases.

Myelin oligodendrocyte glycoprotein (MOG) immunoglobulin G (IgG)-associated disorders (MOGAD), aquaporin 4 (AQP4)-IgG-positive neuromyelitis optica spectrum disorder (AQP4-IgG-NMOSD), and multiple sclerosis (MS) are distinct inflammatory demyelinating disorders of the $\mathrm{CNS}^{1-4}$ Despite some overlapping manifestations, prior studies have highlighted major differences among these disease entities, especially concerning attack severity and clinical course. ${ }^{5-7}$ While AQP4-IgGNMOSD and MOGAD are characterized by severe attacks that typically result in major acute disability (e.g., paraplegia, encephalopathy, blindness) and are frequently accompanied by large MRI T2 lesions in the brain ( $>3 \mathrm{~cm}$ in maximum diameter) or the spinal cord ( $\geq 3$ contiguous vertebral body segments), MS attacks are typically of milder clinical severity and accompanied by smaller lesions on brain/spinal cord MRI, although tumefactive MS lesions occur in some patients. ${ }^{5,6,8-11}$ In the long term, however, the course of these disorders does not generally reflect the severity of the initial attacks: patients with MOGAD frequently have better outcomes than those with AQP4-IgGNMOSD despite similarly severe attacks at nadir, while secondary progressive disability is essentially exclusive to MS despite milder disease attacks in this condition. ${ }^{12-14}$ The exact reasons for these clinical differences are unclear.

Most MRI studies in these 3 disorders have focused on attack lesion location and morphology or global measures of atrophy, while T2 lesion evolution has rarely been assessed., ${ }^{5,6,10,15-17}$ Studying the temporal evolution of different demyelinating lesions on MRI after an initial attack might improve our understanding of differences in disease course, inform diagnosis, and help plan optimal strategies for monitoring disease activity and treatment. We investigated the temporal evolution of demyelinating lesions on MRI after a single brain or spinal cord clinical attack in patients with MOGAD, AQP4-IgG-NMOSD, and MS.

\section{Methods}

\section{Standard Protocol Approvals, Registrations, and Patient Consents}

The study was approved by the institutional review board of the Mayo Clinic. All patients consented to the use of their medical records for research purposes.

\section{Identification of Disease Cohorts}

Three cohorts of patients were retrospectively identified: (1) patients with MOGAD consecutively seen at Mayo Clinic between January 1, 2004, and August 31, 2019 or (2) AQP4-IgG-NMOSD consecutively seen at Mayo Clinic between January 1, 2000, and August 31, 2019; and (3) patients with MS or clinically isolated syndrome from both a clinic-based cohort of patients consecutively seen by a neurologist (E.P.F.) at the Mayo Clinic MS outpatient service from January 1, 2015, to May 30, 2018, and a previously identified population-based cohort (Olmsted County, MN) of prevalent MS cases on December 31, 2011. ${ }^{8}$ Only patients with the characteristic MOGAD, AQP4-IgG-NMOSD, or MS clinical-MRI phenotypes were included as per published international diagnostic criteria. ${ }^{18-20}$ A total of 19 patients were previously reported. ${ }^{5,12,21}$

\section{Inclusion and Exclusion Criteria}

From these cohorts, our inclusion criteria required all of the following: (1) first brain or myelitis attack; (2) available acute brain/spinal cord MRI obtained within 6 weeks of the attack nadir; and (3) available follow-up MRI beyond 6 months after the acute MRI. We excluded those with interval relapses involving the same region as the initial attack (brain or spine) to avoid overlap of MRI abnormalities that could bias assessment of single T2 lesion evolution. Interval relapses affecting different CNS regions were allowed (e.g., optic neuritis after an initial myelitis attack). When multiple acute MRIs were available, the one showing the most extensive CNS abnormalities was chosen for analysis as this was considered to be most representative of attack nadir. In cases with multiple follow-up MRIs, the last MRI before a relapse affecting the same CNS region of the initial attack (if any) or last clinical follow-up was analyzed. In patients who had concomitant brain and myelitis attack at the same time, these were analyzed separately. Clinically isolated optic neuritis attacks with or without MRI evidence of brain or spinal cord asymptomatic involvement were not considered brain attacks. Patients presenting with clinically isolated optic neuritis were not included unless they had a subsequent brain or myelitis attack, which was considered the index attack for analysis. 


\section{Autoantibody Testing}

Serum positivity for AQP4-IgG was determined by live (inhouse fluorescence-activated cell sorting assay) or fixed HEK293 cells transfected with a plasmid encoding AQP4 ( $\mathrm{n}=$ 49 ) or tissue immunofluorescence $(n=2) .{ }^{22}$ Serum positivity for MOG-IgG1 was confirmed by a clinically validated flow cytometry assay utilizing live full-length MOG-transfected HEK 293 cells. ${ }^{5}$ Samples analyzed included those tested at the time of an acute attack and archived stored samples that were re-analyzed.

\section{Clinical Characteristics}

Demographic, laboratory, and clinical data were abstracted (E.S., M.B). Relapses were defined as neurologic disturbances of at least 24 hours duration and occurring after a period of remission of $>1$ month. Disability assessment was performed at nadir of the initial attack and the closest clinical follow-up to the analyzed follow-up MRI using the Expanded Disability Status Scale score (EDSS).

\section{Identification of Index Demyelinating Lesions}

After medical records review, an index lesion was defined as an acute lesion that was anatomically explanatory for the clinical manifestations or, when multiple possible symptomatic acute lesions (e.g., multifocal brain T2 hyperintensities in a patient with encephalopathy) were present, the largest was selected as the index lesion. This index lesion was initially identified for each included attack by 2 neurologists (E.S., E.P.F.) by consensus, and the neuroradiologists were advised of its location before imaging review.

\section{MRI Determination of Lesion Evolution and Lesion Size}

All included acute and follow-up brain and spinal cord MRIs were sorted in random order and independently reviewed by 2 neuroradiologists (S.M., K.N.K.) blinded to the diagnosis and serology status. Lesion resolution or persistence on follow-up MRI was determined, and consensus was reached after discussion when disagreement occurred. To assess evolution in T2 lesion size over time, a single neuroradiologist

Figure 1 Examples of Sagittal and Axial Area Measurements for Spinal Cord and Brain T2 Lesions
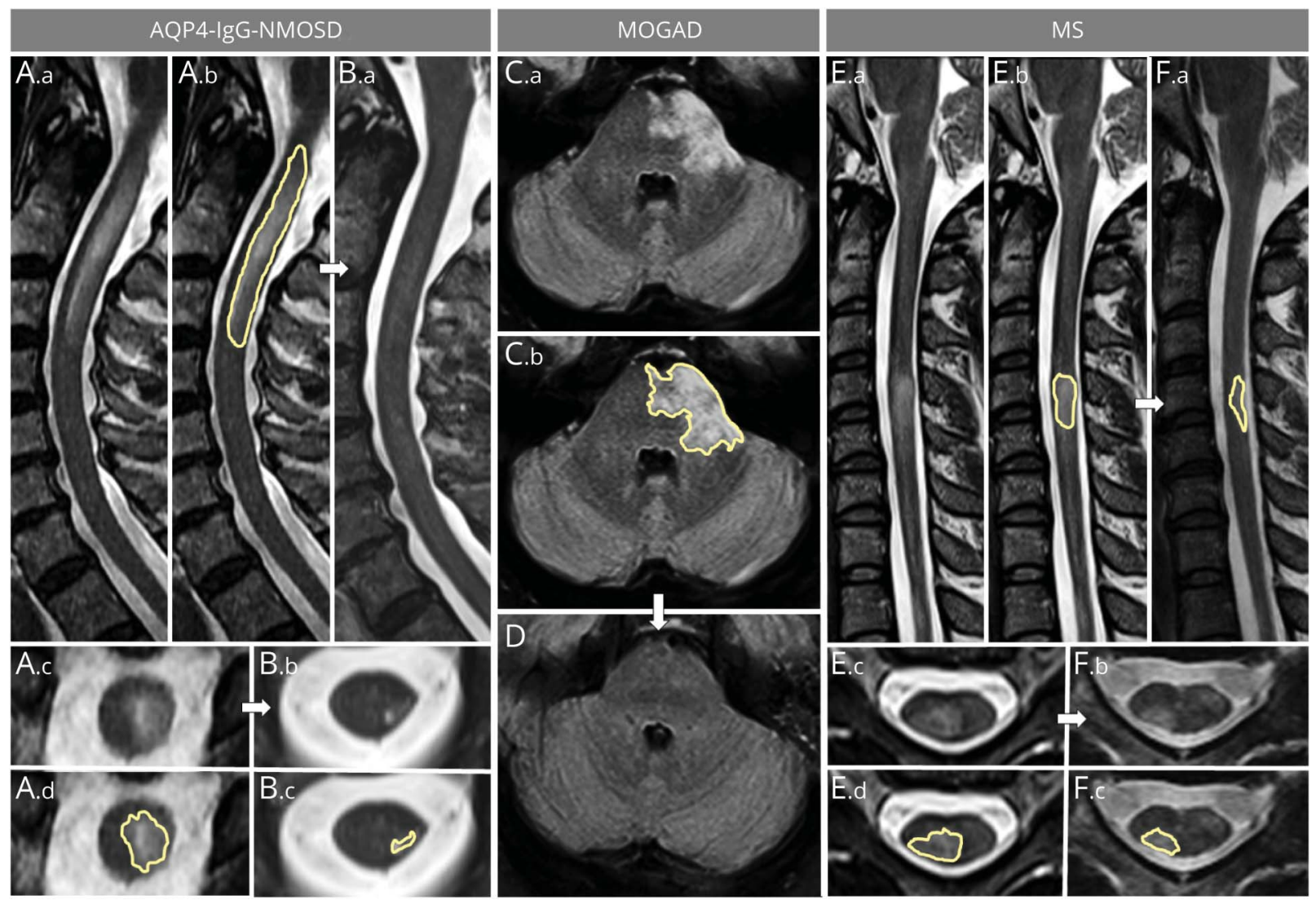

The left part of the figure shows sagittal (A.a, A.b, B.a) and axial (A.c, B.b) T2-weighted images of a longitudinally extensive spinal cord lesion associated with aquaporin 4-immunoglobulin G-positive neuromyelitis optica spectrum disorder (AQP4-IgG-NMOSD) acutely (A) and at follow-up (B). The largest acute axial area of the lesion was manually outlined acutely on sagittal (A.b, yellow outline) and axial (A.d, yellow outline) images and at follow-up (B.C) to determine lesion resolution/reduction. In the center of the figure, an acute brainstem T2-hyperintense lesion associated with myelin oligodendrocyte glycoproteinimmunoglobulin G-associated disorder (MOGAD) is shown on axial fluid-attenuated inversion recovery images (C.a). The largest axial T2 lesion area manually outlined acutely (C.b, yellow outline) resolves to undetectable at follow-up MRI (D). On the right, a multiple sclerosis (MS) myelitis T2-hyperintense lesion is shown (E.a, E.b) with the largest area of the T2-hyperintense lesion outlined acutely on both sagittal (E.b, yellow outline) and axial (E.d, yellow outline) images. Despite being smaller acutely compared to the lesions associated with AQP4-IgG-NMOSD and MOGAD, the MS lesion only shows moderate reduction in size at follow-up, where it is still clearly visible on both sagittal (F.a, yellow outline) and axial (F.b, F.c, yellow outline) images. 
(K.N.K or S.A.M.) manually measured the largest axial area $\left(\mathrm{mm}^{2}\right)$ of the index brain and spinal cord lesions, and largest sagittal area and longest lesion length in contiguous vertebral body segments ( 0.5 or more) for the spinal cord lesions only, in the acute and follow-up MRIs (Figure 1). When inspecting the index lesion location on follow-up MRI examination, the second examination was displayed side-by-side with the index examination and lesion location was coregistered visually prior to characterization and measurement. Images were obtained on a variety of different scanners from both outside centers and Mayo Clinic and were analyzed by using QREADS Clinical Image Viewer 5.10.8. Additional MRI parameters collected included (1) frequency of patients with $>15$ T2-hyperintense lesions on T2/fluid-attenuated inversion recovery (FLAIR) images; (2) number of infratentorial T2 lesions on T2/FLAIR images; (3) number of spinal cord T2-hyperintense lesions on T2-weighted images; (4) presence of abnormally enhancing lesions on postgadolinium T1-weighted images acutely and at follow-up; and (5) presence of residual lesional T1 hypointensity in the brain or spinal cord parenchyma at follow-up.

\section{Statistical Analysis}

Continuous and categorical variables were reported as median (range) and number (\%), respectively. Comparisons across the 3 groups were performed by using the Kruskal-Wallis test for continuous variables and Fisher exact test for categorical variables. Pairwise comparisons were performed by using the Wilcoxon rank sum test for continuous variables and Fisher exact test for categorical variables. Potential associations were explored by univariate logistic regression. A $p$ value $<0.05$ was considered statistically significant (JMP Pro 14.1.0). Graphs were created with Microsoft Power Point 2010.

\section{Data Availability}

Anonymized data used for this study are available upon reasonable request from the corresponding author.

\section{Results}

A total of 156 patients were included (MOGAD, 38; AQP4IgG-NMOSD, 51; MS, 67) with a total of 172 attacks (brain, 81; myelitis, 91). In 16 patients (MOGAD, 8; AQP4-IgGNMOSD, 4; MS, 4) both a brain and a myelitis attack were analyzed, which occurred within the same month in 14 . Their demographics, laboratory, and clinical characteristics are summarized in Table 1. In the MS cohort, the clinical phenotypes at last follow-up were as follows: relapsing-remitting MS, $n=63$; single attack progressive $\mathrm{MS}, \mathrm{n}=2$; and clinically isolated syndrome, $\mathrm{n}=2$. Among AQP4-IgG-NMOSD and MOGAD, no patient had developed a progressive course at last follow-up.

All patients with MOGAD tested negative for AQP4-IgG, while in the AQP4-IgG-NMOSD cohort, 38 of 51 (75\%) were tested for MOG-IgG, uniformly with negative results. In the MS cohort, $36(54 \%)$ and 31 (46\%) of 67 were tested for AQP4-IgG and MOG-IgG, respectively, all of whom were seronegative, including the 2 patients with clinically isolated syndrome who tested negative for both antibodies.

\section{Description of the Attacks}

The brain symptoms accompanying the index lesions were encephalopathy or focal symptoms attributable to supratentorial brain regions in 12 (MOGAD, 7; AQP4-IgGNMOSD, 0 ; MS, 5; $p=0.003)$, brainstem/cerebellar syndrome in 56 (MOGAD, 10; AQP4-IgG-NMOSD, 18; MS, $28 ; p=0.1$ ), and combinations thereof in 13 (MOGAD, 1 ; AQP4-IgG-NMOSD, 3; MS, 9; $p=0.4$ ). In patients with an initial myelitis attack, spinal cord MRI demonstrated cervical involvement in 31 (MOGAD, 6; AQP4-IgG-NMOSD, 9; MS, 16; $p=0.02$ ), thoracic involvement in 21 (MOGAD, 7; AQP4-IgG-NMOSD, 11; MS, 3; $p=0.1$ ), or involvement of both regions in 39 (MOGAD, 15; AQP4-IgG-NMOSD, 14; MS, $10 ; p=0.36$ ). In patients with MOGAD, the included attacks occurred as part of an acute disseminated encephalomyelitis or with MRI evidence of multifocal CNS involvement without encephalopathy in $22 / 38(58 \%)$ or as isolated brain $(4 / 38[11 \%])$ or myelitis $(12 / 38[32 \%])$ attacks with or without concomitant optic neuritis.

\section{Interval Treatment}

Acute immunotherapy (within 6 weeks from the index attack nadir) was more commonly a monotherapy of IV or oral corticosteroids in the MS cohort (43/46 [93\%]) compared to the MOGAD (23/38 [61\%]) and AQP4-IgG-NMOSD (29/ $46[63 \%])$ groups $(p<0.001)$, although single-agent treatments with IV immunoglobulins (IVIg; 2 patients with MOGAD) or plasma exchange (PLEX; 1 patient with AQP4IgG-NMOSD) were also administered. In addition to corticosteroids, 1 or more adjunctive acute treatments utilized in each disorder included the following: MS (PLEX, 3); AQP4IgG-NMOSD (PLEX, 16; IVIg, 2); and MOGAD (PLEX, 7; IVIg, 6). After the acute phase, most of the patients in the 3 groups received different maintenance immunomodulating or immunosuppressive agents, as detailed in Table 1.

\section{Other CNS Interval Relapses}

Relapses in other regions of the nervous system were allowed between the attack MRI and the follow-up MRI. This included myelitis interval relapses in patients with initial brain attacks in 8/21 (38\%) patients with AQP4-IgG-NMOSD, 9/ 18 (50\%) patients with MOGAD, and 16/42 (38\%) patients with MS $(p=0.83)$, and interval brain or optic neuritis relapses in patients with initial myelitis attacks in 10/34 (29\%) patients with AQP4-IgG-NMOSD, 13/28 (46\%) patients with MOGAD, and 5/29 (17\%) patients with MS $(p=0.07)$.

\section{MRI Lesion Evolution}

The acute and follow-up MRI characteristics for the included attacks are summarized and compared in Tables 2 and 3; representative examples of brain and spinal cord MRI lesion evolution are shown in Figures 1-3. Graphs of the main differences in MRI lesion evolution measures for the 3 disease groups are included in Figure 4. In patients with brain attacks, the median (range) number of total available follow-up brain MRIs per patient before a brain relapse or last clinical contact was 3 (1-14): AQP4-IgG-NMOSD, 3 (1-10); MOGAD, 3 
Table 1 Demographics and Clinical Characteristics of the 156 Included Patients

\begin{tabular}{|c|c|c|c|c|c|c|}
\hline & AQP4-IgG-NMOSD & MOGAD & MS & $\begin{array}{l}\text { p AQP4-IgG-NMOSD } \\
\text { vs MOGAD }\end{array}$ & $\begin{array}{l}p \text { MOGAD } \\
\text { vs MS }\end{array}$ & $\begin{array}{l}\text { p AQP4-IgG-NMOSD } \\
\text { vs MS }\end{array}$ \\
\hline Patients & 51 & 38 & 67 & - & - & - \\
\hline Age at initial attack, $y$, mean (range) & $53(10-78)$ & $25(2-74)$ & $37(16-61)$ & $<0.0001$ & 0.002 & $<0.0001$ \\
\hline Pediatric (age $<18$ y) & $2 / 51(4)$ & $14 / 38(37)$ & $2 / 67(3)$ & 0.0001 & $<0.0001$ & 1.0 \\
\hline Female sex & $41 / 51(80)$ & $17 / 38(45)$ & $51 / 67(76)$ & 0.001 & 0.002 & 0.7 \\
\hline CSF OCB $(\geq 2)$ & $4 / 25(16)$ & $3 / 23(13)$ & $31 / 36(86)$ & 1.0 & $<0.0001$ & $<0.0001$ \\
\hline Total number of attacks & 55 & 46 & 71 & - & - & - \\
\hline Brain attacks & 21 & 18 & 42 & - & - & - \\
\hline Myelitis attacks & 34 & 28 & 29 & - & - & - \\
\hline Initial attack as disease presentation ${ }^{a}$ & $30 / 51(59)$ & $31 / 38(82)$ & $41 / 67(61)$ & 0.04 & 0.05 & 0.9 \\
\hline Median EDSS at attack nadir (range) & $6(2-9.5)$ & $6(3-9)$ & $3(0-7)$ & 0.7 & $<0.0001$ & $<0.0001$ \\
\hline Acute treatment & $46 / 50(92)$ & $38 / 38(100)$ & $46 / 67(69)$ & 0.3 & $<0.0001$ & 0.001 \\
\hline Corticosteroids & 45 & 36 & 46 & - & - & - \\
\hline PLEX & 17 & 7 & 3 & - & - & - \\
\hline IVlg & 2 & 8 & 0 & - & - & - \\
\hline Maintenance therapy & $47 / 51(92)$ & $30 / 38(79)$ & $51 / 67(76)$ & 0.1 & 0.8 & 0.03 \\
\hline Corticosteroids & 32 & 23 & 9 & - & - & - \\
\hline MMF, AZA & 32 & 13 & 1 & - & - & - \\
\hline Anti-CD20 ${ }^{b}$ & 21 & 7 & 3 & - & - & - \\
\hline IFN- $\beta$, GA & 2 & 4 & 38 & - & - & - \\
\hline DMF, FGD, NTZ, TER & 1 & 1 & 25 & - & - & - \\
\hline Other & 12 & 4 & 2 & - & - & - \\
\hline Median EDSS score at last follow-up (range) & $3(0-8)$ & $1(0-7)$ & $1(0-7)$ & $<0.0001$ & 0.5 & $<0.0001$ \\
\hline Median EDSS score $\geq 6$ at last follow-up & $10 / 49(20)$ & $2 / 38(5)$ & $4 / 65(6)$ & 0.06 & 1.0 & 0.04 \\
\hline Monophasic disease course at last follow-up & $14 / 51(27)$ & $13 / 38(34)$ & $18 / 67(27)$ & 0.6 & 0.5 & 1.0 \\
\hline Median clinical follow-up duration, mo & $30(4-143)$ & $19(4-121)$ & $31(6-201)$ & 0.3 & 0.02 & 0.3 \\
\hline
\end{tabular}

Abbreviations: AQP4-IgG-NMOSD = aquaporin 4-immunoglobulin G-positive neuromyelitis optica spectrum disorder; $A Z A=$ azathioprine; DMF = dimethyl fumarate; EDSS = Expanded Disability Status Scale; FGD = fingolimod; GA = glatiramer acetate; IFN = Interferon; IVIg = IV immunoglobulin; MMF = mycophenolate mofetil; MOGAD = myelin oligodendrocyte glycoprotein-immunoglobulin G-associated disorder; MS = multiple sclerosis; NTZ = natalizumab; OCB = oligoclonal bands; PLEX = plasma exchange; TER = teriflunomide.

Results are presented as number (\%) unless specified otherwise.

${ }^{a}$ In 54 patients, the index attack was preceded by other disease-related CNS manifestations including optic neuritis, brain/brainstem manifestations (in patients with myelitis index attack), or myelitis (in patients with brain index attack).

${ }^{b}$ Rituximab or ocrelizumab.

(1-13); MS, 3.5 (1-14). In patients with myelitis attacks, the median (range) number of available follow-up spinal cord MRIs per patient before a myelitis relapse or last clinical contact was 2 (1-8): AQP4-IgG-NMOSD, 2 (1-7); MOGAD, 3 (1-7); MS, 2 (1-8).

\section{Index T2 Lesion Resolution}

Complete resolution of the brain index lesion, with or without normalization of the entire brain MRI, was observed in $72 \%$ of patients with MOGAD, $17 \%$ of patients with MS, and $14 \%$ of patients with AQP4-IgG-NMOSD $(p<0.001)$ (Table 2). In the AQP4-IgG-NMOSD and MS groups, all index lesions that resolved completely were infratentorial, while in the MOGAD group, they were both supratentorial $(\mathrm{n}=6)$ and infratentorial $(\mathrm{n}=7)$.

Complete resolution of the myelitis index lesion was observed in $79 \%$ of patients with MOGAD, $0 \%$ of patients with MS, and $0 \%$ of patients with AQP4-IgG-NMOSD $(p<0.001)$ (Table 3). In patients with a myelitis attack, resolution of the 
Table 2 MRI Characteristics of Brain Attacks Acutely and at Follow-up

\begin{tabular}{|c|c|c|c|c|c|c|}
\hline & $\begin{array}{l}\text { AQP4-IgG- } \\
\text { NMOSD }\end{array}$ & MOGAD & MS & $\begin{array}{l}p \text { AQP4-IgG- } \\
\text { NMOSD vS } \\
\text { MOGAD }\end{array}$ & $\begin{array}{l}p \text { MOGAD } \\
\text { vS MS }\end{array}$ & $\begin{array}{l}p \text { AQP4-IgG- } \\
\text { NMOSD vS MS }\end{array}$ \\
\hline Brain attacks, $\mathrm{n}$ & 21 & 18 & 42 & - & - & - \\
\hline Number of brain MRI T2 lesions $>15$ on attack MRI & $9 / 21(43)$ & $10 / 18(56)$ & $20 / 42(48)$ & 0.5 & 0.8 & 0.8 \\
\hline Infratentorial T2 lesions on attack MRI & $19 / 21(90)$ & $14 / 18(78)$ & $36 / 42(86)$ & 0.4 & 0.5 & 0.7 \\
\hline $\begin{array}{l}\text { Axial area of the index } \mathrm{T} 2 \text { lesion in } \mathrm{mm}^{2} \text { on attack } \\
\text { MRI }\end{array}$ & $167(14-635)$ & $213(66-873)$ & $58(8-754)$ & 0.1 & $<0.001$ & 0.01 \\
\hline Gadolinium enhancement on attack MRI & $13 / 20(65)$ & $8 / 18(44)$ & $29 / 39(74)$ & 0.3 & 0.03 & 0.4 \\
\hline Resolution of the index T2 lesion at follow-up & $3 / 21(14)$ & $13 / 18(72)$ & $7 / 42(17)$ & $<0.001$ & $<0.001$ & 1.0 \\
\hline Normal brain MRI at follow-up & $2 / 21(10)$ & $7 / 18(39)$ & $2 / 42(5)$ & 0.05 & 0.002 & 0.6 \\
\hline $\begin{array}{l}\text { Residual axial area of the index } \mathrm{T} 2 \text { lesion in } \mathrm{mm}^{2} \text { on } \\
\text { follow-up MRI }\end{array}$ & $26(0-247)$ & $0(0-114)$ & $13(0-248)$ & 0.002 & 0.01 & 0.1 \\
\hline $\begin{array}{l}\text { Delta axial area reduction of the index } \mathrm{T} 2 \text { lesion in } \\
\mathrm{mm}^{2} \text { from acute } \mathrm{MRI}^{\mathrm{a}}\end{array}$ & $104(0.7-597)$ & $213(55-873)$ & $36(0-506)$ & 0.02 & $<0.001$ & 0.01 \\
\hline $\begin{array}{l}\text { \% Axial area reduction of the index } \mathrm{T} 2 \text { lesion from } \\
\text { acute } \mathrm{MRI}^{\mathrm{b}}\end{array}$ & $78(4-100)$ & $100(63-100)$ & $75(0-100)$ & $<0.001$ & $<0.001$ & 0.8 \\
\hline Persistent enhancement on follow-up MRI & $0 / 13(0)$ & $0 / 8(0)$ & $0 / 29(0)$ & - & - & - \\
\hline Residual T1 hypointensity for the index lesion & $9 / 21(43)$ & $0 / 18(0)$ & $20 / 42(48)$ & 0.002 & $<0.001$ & 0.8 \\
\hline Any residual brain T1 hypointensity at follow-up MRI & $12 / 21(57)$ & $3 / 18(17)$ & $35 / 42(83)$ & 0.02 & $<0.001$ & 0.03 \\
\hline Time interval from acute to follow up MRI, mo & $43(6-143)$ & $21(10-109)$ & $37(7-192)$ & 0.9 & 0.3 & 0.5 \\
\hline
\end{tabular}

Abbreviations AQP4-IgG NMOSD = aquaporin 4-immunoglobulin G-positive neuromyelitis optica spectrum disorder; MOGAD = myelin oligodendrocyte glycoprotein-immunoglobulin G-associated disorder; MS = multiple sclerosis.

Results are presented as median (range) or $\mathrm{n}(\%)$.

a Delta area reductions were calculated for each patient as (acute area - follow-up area).

b \% Area reductions were calculated as (acute area - follow-up area)/acute area.

index lesion always coincided with normalization of the spinal cord MRI at follow-up.

\section{MRI Normalization of T2 Signal Abnormalities}

A complete resolution of all the specific T2 abnormalities at MRI follow-up was more common with MOGAD for both brain (MOGAD, 39\%; AQP4-IgG-NMOSD, 10\%; MS, 5\%) and spinal cord (MOGAD, 79\%; AQP4-IgG-NMOSD, 0\%; MS, 0\%) attacks (Tables 2 and 3). By reviewing all available follow-up MRIs (including those obtained before 6 months), the median (range) time in months from acute to first available normal brain MRI in those in whom T2 lesions resolved were as follows: MOGAD, 19 (1-96); AQP4-IgG-NMOSD, 26 (18-95); MS, 11 (10-13). In patients with MOGAD with a myelitis attack, the median (range) time to first normal follow-up MRI was 9 months (1-96).

\section{Determinants of Lesion Resolution in MOGAD}

By using logistic regression to predict $\mathrm{T} 2$ lesion resolution in patients with MOGAD, no significant association was found with time interval between acute and follow-up MRI, sex, acute lesion area, abnormal gadolinium enhancement on acute MRI, interval disease relapses in other CNS regions, cervical vs thoracic spinal cord location (in patients with myelitis only),
MOGAD phenotype (acute disseminated encephalomyelitis/ multifocal CNS involvement vs other), monophasic vs relapsing course of the disease, administration of acute treatment and its type (monotherapy vs multiple agents), or commencement of long-term immunosuppressive therapy and its types (anti-CD20 vs oral immunosuppressants [azathioprine or mycophenolate mofetil] vs MS disease-modifying agents). Moreover, there was no difference between pediatric (age $<18$ years) and adult patients with MOGAD in the frequency of brain index lesion resolution $(7 / 11[64 \%]$ vs $6 / 7$ [86\%]; $p=$ 0.6), brain MRI normalization (4/11 [36\%] vs 3/7 [43\%]; $p=$ $1.0)$, and spinal cord MRI normalization (5/8 [63\%] vs $17 / 20$ [85\%]; $p=0.3)$. Lastly, for those in whom repeated MOG-IgG testing was available, the frequency of lesion resolution did not differ significantly for transient (i.e., testing negative at least 6 months after the initial MOG-IgG positivity) vs persistent $(\geq 6$ months) antibody positivity and brain (3/4 [75\%] vs $8 / 10$ $[80 \%] ; p=1.0)$ or myelitis $(3 / 6[50 \%]$ vs $10 / 12[83 \%] ; p=$ 0.27 ) attacks, respectively.

\section{Clinical Characteristics of Patients With MRI Normalization}

In the MOGAD group, the median EDSS at last clinical follow-up was similar in those who showed a normal MRI 
Table 3 MRI Characteristics of Myelitis Attacks Acutely and at Last Follow-up

\begin{tabular}{|c|c|c|c|c|c|c|}
\hline & $\begin{array}{l}\text { AQP4-IgG- } \\
\text { NMOSD }\end{array}$ & MOGAD & MS & $\begin{array}{l}\text { p AQP4-IgG- } \\
\text { NMOSD vs } \\
\text { MOGAD }\end{array}$ & $\begin{array}{l}p \\
\text { MOGAD } \\
\text { vs MS }\end{array}$ & $\begin{array}{l}\text { p AQP4-IgG- } \\
\text { NMOSD vs MS }\end{array}$ \\
\hline Spinal cord attacks, $n$ & 34 & 28 & 29 & - & - & - \\
\hline Number of spinal cord T2 lesions on attack MRI & $1(1-5)$ & $1(1-4)$ & $2(1-6)$ & 0.046 & 0.2 & 0.001 \\
\hline $\begin{array}{l}\text { Sagittal length of the index T2 lesion in VBS on } \\
\text { attack MRI }\end{array}$ & $6.25(1-19)$ & $4.5(0.5-19)$ & $1(0.5-2)$ & 0.2 & $<0.001$ & $<0.001$ \\
\hline $\begin{array}{l}\text { Sagittal area of the index } \mathrm{T} 2 \text { lesion in } \mathrm{mm}^{2} \text { on attack } \\
\text { MRI }\end{array}$ & $437(46-2,120)$ & $279(17-888)$ & $64(19-229)$ & 0.05 & $<0.001$ & $<0.001$ \\
\hline $\begin{array}{l}\text { Axial area of the index } \mathrm{T} 2 \text { lesion in } \mathrm{mm}^{2} \text { on attack } \\
\text { MRI }\end{array}$ & $39(9-118)$ & $45(4-100)$ & $21(9-62)$ & 0.8 & 0.005 & $<0.001$ \\
\hline Gadolinium enhancement on attack MRI & $32 / 34(94)$ & $15 / 28(54)$ & $25 / 28(89)$ & $<0.001$ & 0.01 & 0.7 \\
\hline Resolution of the index T2 lesion on follow-up MRI & $0 / 34(0)$ & $22 / 28(79)$ & $0 / 29(0)$ & $<0.001$ & $<0.001$ & - \\
\hline Normal spinal cord MRI at follow-up & $0 / 34(0)$ & $22 / 28(79)$ & $0 / 29(0)$ & $<0.001$ & $<0.001$ & - \\
\hline $\begin{array}{l}\text { Residual sagittal length of the index T2 lesion in VBS } \\
\text { on follow-up MRI }\end{array}$ & $2.5(0-7)$ & $0(0-7)$ & $0.5(0-2)$ & $<0.001$ & $<0.001$ & $<0.001$ \\
\hline $\begin{array}{l}\text { Residual sagittal area of the index } \mathrm{T} 2 \text { lesion in } \mathrm{mm}^{2} \\
\text { on follow-up MRI }\end{array}$ & $87(0-513)$ & $0(0-237)$ & $34(5-229)$ & $<0.001$ & 0.001 & $<0.001$ \\
\hline $\begin{array}{l}\text { Delta sagittal area reduction of the index } \mathrm{T} 2 \text { lesion } \\
\text { in } \mathrm{mm}^{2} \text { from acute } \mathrm{MRI}^{\mathrm{a}}\end{array}$ & $309(0-1885)$ & $262(0-888)$ & $23(0-152)$ & 0.4 & $<0.001$ & $<0.001$ \\
\hline 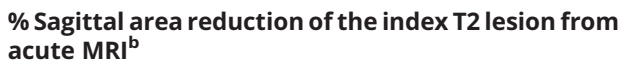 & $74(0-100)$ & $100(0-100)$ & $39(0-94)$ & $<0.001$ & $<0.001$ & $<0.001$ \\
\hline $\begin{array}{l}\text { Residual axial area of the index } \mathrm{T} 2 \text { lesion in } \mathrm{mm}^{2} \text { on } \\
\text { follow-up MRI }\end{array}$ & $9(0-30)$ & $0(0-60)$ & $10(0-32)$ & $<0.001$ & $<0.001$ & 0.6 \\
\hline $\begin{array}{l}\text { Delta axial area reduction of the index } \mathrm{T} 2 \text { lesion in } \\
\mathrm{mm}^{2} \text { from acute } \mathrm{MRI}^{\mathrm{a}}\end{array}$ & $23(1-118)$ & $34(4-100)$ & $10(0-48)$ & 0.3 & $<0.001$ & $<0.001$ \\
\hline $\begin{array}{l}\% \text { Axial area reduction of the index } \mathrm{T} 2 \text { lesion from } \\
\text { acute } \mathrm{MRI}^{\mathbf{b}}\end{array}$ & $71(0-100)$ & $100(33-100)$ & $53(0-100)$ & $<0.001$ & $<0.001$ & 0.001 \\
\hline Persistent enhancement on follow-up MRI & $0 / 32(0)$ & $0 / 15(0)$ & $0 / 25(0)$ & - & - & - \\
\hline Residual T1 hypointensity for the index lesion & $3 / 34(9)$ & $0 / 28(0)$ & $1 / 29(3)$ & 0.2 & 1.0 & 0.6 \\
\hline $\begin{array}{l}\text { Any residual spinal cord T1 hypointensity at follow- } \\
\text { up MRI }\end{array}$ & $3 / 34(9)$ & $0 / 28(0)$ & $1 / 29(3)$ & 0.2 & 1.0 & 0.6 \\
\hline Time interval from acute to follow-up MRI, mo & $31(6-138)$ & $15(7-104)$ & $26(6-201)$ & 0.1 & 0.04 & 0.6 \\
\hline
\end{tabular}

Abbreviations AQP4-IgG NMOSD = aquaporin 4-immunoglobulin G-positive neuromyelitis optica spectrum disorder; MOGAD = myelin oligodendrocyte glycoprotein-immunoglobulin G-associated disorder; MS = multiple sclerosis; VBS = vertebral body segments.

Results are presented as median (range) or $\mathrm{n}(\%)$.

a Delta area reductions were calculated for each patient as (acute area - follow-up area).

b\% Area reductions were calculated as (acute area - follow-up area)/acute area.

(brain, EDSS 1 [range, 0-2]; myelitis, EDSS 1 [0-6.5]) vs those who did not (brain, EDSS 1 [range, 0-3.5]; myelitis, EDSS 0.5 [range, $0-7])(p \geq 0.6$ for all comparisons). The frequencies of interval relapses were similar. Three patients with isolated MOGAD myelitis (and no other disease relapses) had residual symptoms of spinal cord dysfunction at the time of the normal follow-up MRI, including subjective sensory disturbances ( $\mathrm{n}=3$ : itching, burning, tingling), bladder dysfunction ( $\mathrm{n}=2$ : increased urinary frequency, urinary hesitancy), and mild gait instability $(\mathrm{n}=1)$. Also, one patient with clinically isolated syndrome of brainstem demyelination resulting in acute bilateral internuclear ophthalmoplegia had residual intermittent diplopia and gazeevoked nystagmus at last follow-up despite brain MRI normalization. All other patients with normalization of the MRI at follow-up either had no residual disability at last follow-up or a residual clinical deficit explained by other attacks.

\section{Reductions in Size of Index T2 Lesions}

In patients with brain attacks, the median axial area reduction for the index lesions was higher in MOGAD $\left(213 \mathrm{~mm}^{2}\right.$ [range, 55-873]) compared to AQP4-IgG-NMOSD (104 $\mathrm{mm}^{2}$ [range, 0.7-597]) and MS (36 $\mathrm{mm}^{2}$ [range, 0-506]) $(p<$ 0.001 ), although no significant difference was observed 
Figure 2 Brain MRI T2 Lesion Evolution in Aquaporin 4-Immunoglobulin G-Positive Neuromyelitis Optica Spectrum Disorder (AQP4-IgG-NMOSD), Myelin Oligodendrocyte Glycoprotein-Immunoglobulin G-Associated Disorder (MOGAD), and Multiple Sclerosis (MS)
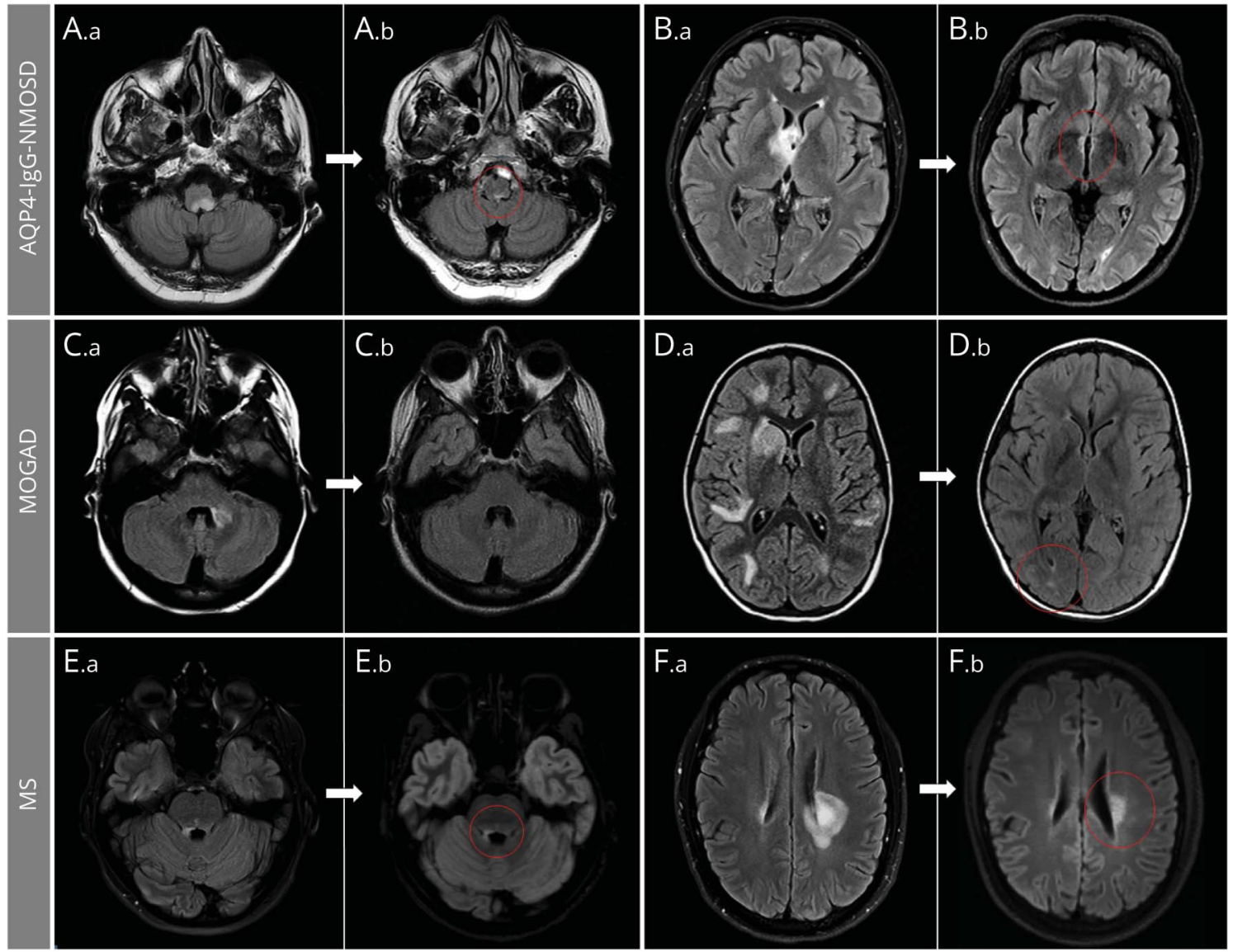

Axial fluid-attenuated inversion recovery infratentorial (A, C, E) and supratentorial (B, D, F) brain MRI are shown. In patients with AQP4-IgG-NMOSD (A, B), large acute lesions (A.a, B.a) commonly show a prominent reduction of their initial size but rarely resolve completely, typically leaving tiny residual abnormalities (A.b, B.b; red circles). In patients with MOGAD (C, D), most large acute T2-hyperintense lesions (C.a, D.a) resolve to undetectable (C.b) at follow-up MRI, although some acute T2 lesions can leave some (often nonspecific) small foci of T2 hyperintensity (D.b; red circle). In patients with MS (E, F), acute T2hyperintense lesions are relatively smaller (E.a, F.a) and typically show moderate reduction in size at follow-up, while remaining clearly detectable (E.b, F.b; red circles).

between patients with AQP4-IgG-NMOSD and MS in the median relative $\%$ reduction from acute MRI (Table 2). In the spinal cord, the median index lesion area reduction was similar in MOGAD and AQP4-IgG-NMOSD on both sagittal (262 [range, $0-888]$ vs 309 [range, $0-1885] \mathrm{mm}^{2} ; p=0.4$ ) and axial (34 [range, $4-100$ ] vs 23 [range, $1-118] \mathrm{mm}^{2} ; p=0.3$ ) images, although the median relative \% reduction in size compared to the acute MRI was higher in the MOGAD group (Table 3 ). In the MS group, both the median axial area reduction in $\mathrm{mm}^{2}$ and the relative $\% \mathrm{~T} 2$ lesion reduction from the acute MRI were lower compared to the MOGAD and AQP4-IgG-NMOSD groups ( $p<0.001$ for all comparisons; Table 3$)$.

\section{Other MRI Measures}

None of those with enhancing lesions acutely showed persistent enhancement at follow-up in any of the 3 groups (Tables 2 and 3). Residual T1 hypointensity of the index brain lesion and of all brain lesions was lowest with MOGAD vs
AQP4-IgG-NMOSD and MS (Table 2) but was very infrequently detected in the spine across all groups (Table 3 ).

\section{Discussion}

We identified clear differences in T2 lesion evolution between MOGAD, AQP4-IgG-NMOSD, and MS that may help define their different disease courses, inform the diagnosis, and help plan future clinical trials. Demyelinating T2 lesions in MOGAD tend to resolve completely at follow-up despite their large size acutely and initial accompanying severe clinical impairment, which is in line with the favorable long-term outcome reported in most patients. ${ }^{12} \mathrm{~T} 2$ lesions associated with AQP4-IgG are similar to those observed in MOGAD in terms of accompanying clinical severity and size but rarely resolve completely and despite a large reduction in size (generally $60 \%-80 \%$ from the acute MRI) small regions of residual T2 hyperintensity are usually evident. This presumed scarring on MRI, along with the 
Figure 3 Spinal Cord MRI Lesion Evolution in Aquaporin 4-Immunoglobulin G-Positive Neuromyelitis Optica Spectrum Disorder (AQP4-IgG-NMOSD), Myelin Oligodendrocyte Glycoprotein-Immunoglobulin G-Associated Disorder (MOGAD), and Multiple Sclerosis (MS)
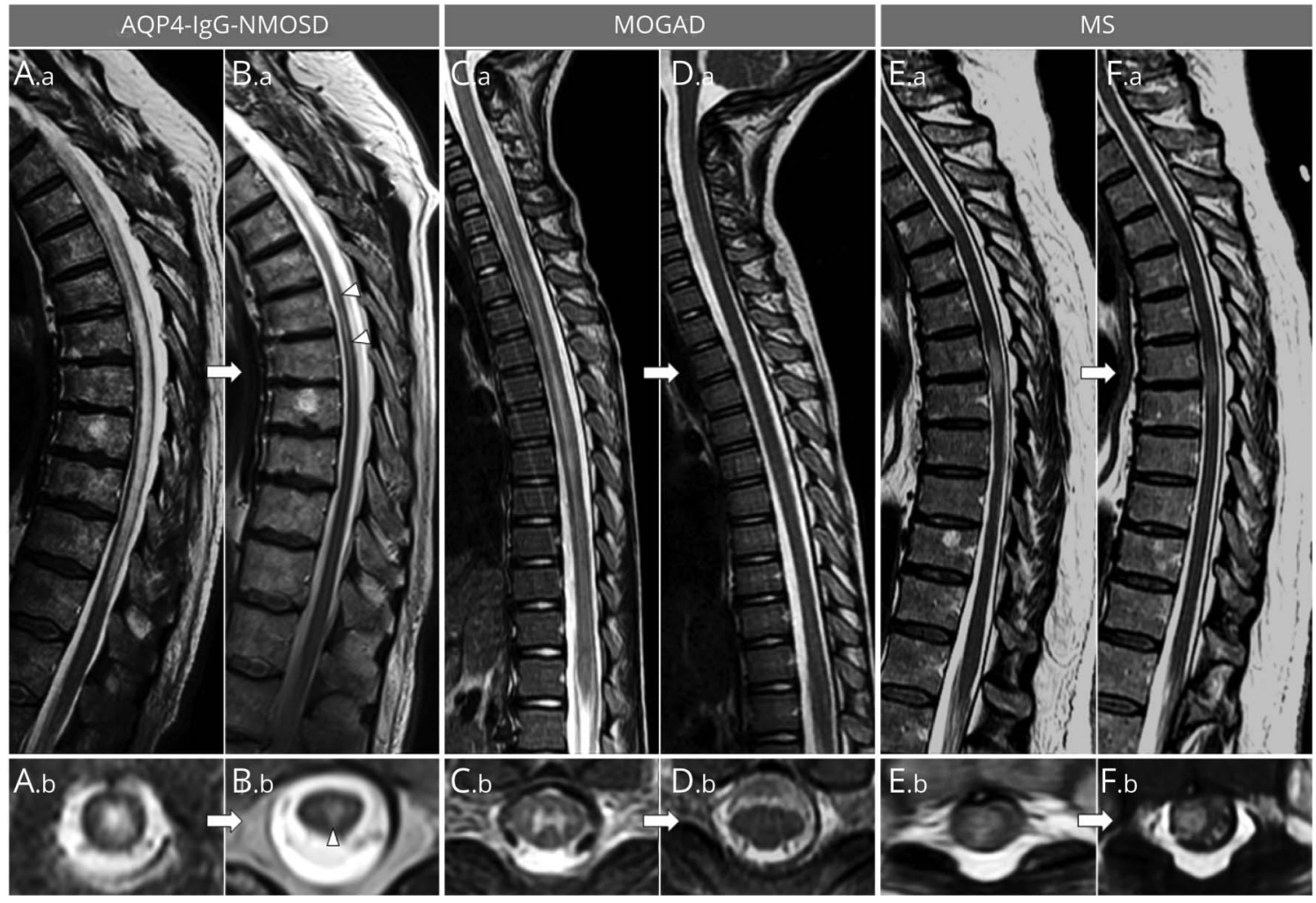

Sagittal (A.a, B.a, C.a, D.a, E.a, F.a) and axial (A.b, B.b, C.b, D.b, E.b, F.b) thoracic spinal cord T2-weighted images are shown. In patients with myelitis, T2hyperintense lesions associated with AQP4-IgG-NMOSD, longitudinally extensive lesions with swelling of the spinal cord (A.a) and holocord involvement acutely (A.b [T7 vertebral body level]), frequently show a dramatic reduction in size at follow-up MRI but remain detectable, often accompanied by focal lesional spinal cord atrophy (B.a, B.b [T7 vertebral body level]). In patients with MOGAD myelitis, the acute ventral-predominant long T2 lesion extending to the conus (C.a) is gray matter restricted axially (C.b [T3 vertebral body level]) and resolved completely at follow-up MRI (D.a, D.b [T3 vertebral body level]). In patients with MS, the typically short acute myelitis T2-hyperintense lesion (E.a, E.b [T6-T7 vertebral body level]) often only has modest reduction in size at follow-up MRI (F.a, F.b [T6-T7 vertebral body level]), at which time it can be better appreciated to involve the periphery (right lateral columns) of the spinal cord on axial images (F.b [T6-T7 vertebral body level]).

greater associated spinal cord atrophy compared to MOGAD described in prior studies, ${ }^{23}$ are consistent with the incomplete recovery and stepwise attack-related disability accumulation reported in AQP4-IgG-NMOSD. ${ }^{24}$ MS lesions are characterized by smaller size and less severe attacks acutely but only show moderate reduction in size (generally 30\%-70\% from the acute MRI) and rarely resolve completely despite resolution of clinical features. We speculate that persistence of these T2 lesions, particularly larger lesions in critical locations, over time may represent a nidus for secondary disability progression and the tendency of some MS lesions to remain chronically active between relapses (smoldering MS lesions) could support this hypothesis. ${ }^{25,26}$ Moreover, reports of progressive disability attributable to a single persistent large T2 lesion in an eloquent location (such as the spinal cord lateral columns) that becomes focally atrophic over time is also consistent with this proposition. ${ }^{27-29}$

While the persistence of MS lesions over time is wellrecognized, lesion evolution in MOGAD and AQP4-IgG-
NMOSD is much less well studied and mostly analyzed in subsets of prior studies. In a cohort of children with demyelinating CNS disorders, normalization of spinal cord MRI after the acute phase was observed in 6/8 (75\%) MOGAD and 0/4 (0\%) AQP4-IgG-NMOSD cases with longitudinally extensive transverse myelitis. ${ }^{30}$ Complete resolution of brain MRI abnormalities was also reported in 25/42 (60\%) children with MOGAD after a median of 4 years, but only $20 / 109$ (18\%) seronegative patients with pediatric CNS demyelination (with approximately half of the patients having an MS phenotype, none of whom showed lesion resolution). ${ }^{31}$ We have previously reported patients with MOGAD with long follow-up (median of 14 years) and highly relapsing course with a normal follow-up MRI of the brain and spinal cord observed in 18/25 (72\%) and 6/6 (100\%) patients, respectively. ${ }^{12}$ Also, a recent series of patients with MOGAD with brainstem or cerebellar attacks from our group showed lesion resolution at $\geq 6$ months observed in 6/9 (67\%) patients. ${ }^{21}$ Accepting differences in study design and follow-up durations, these numbers are in 

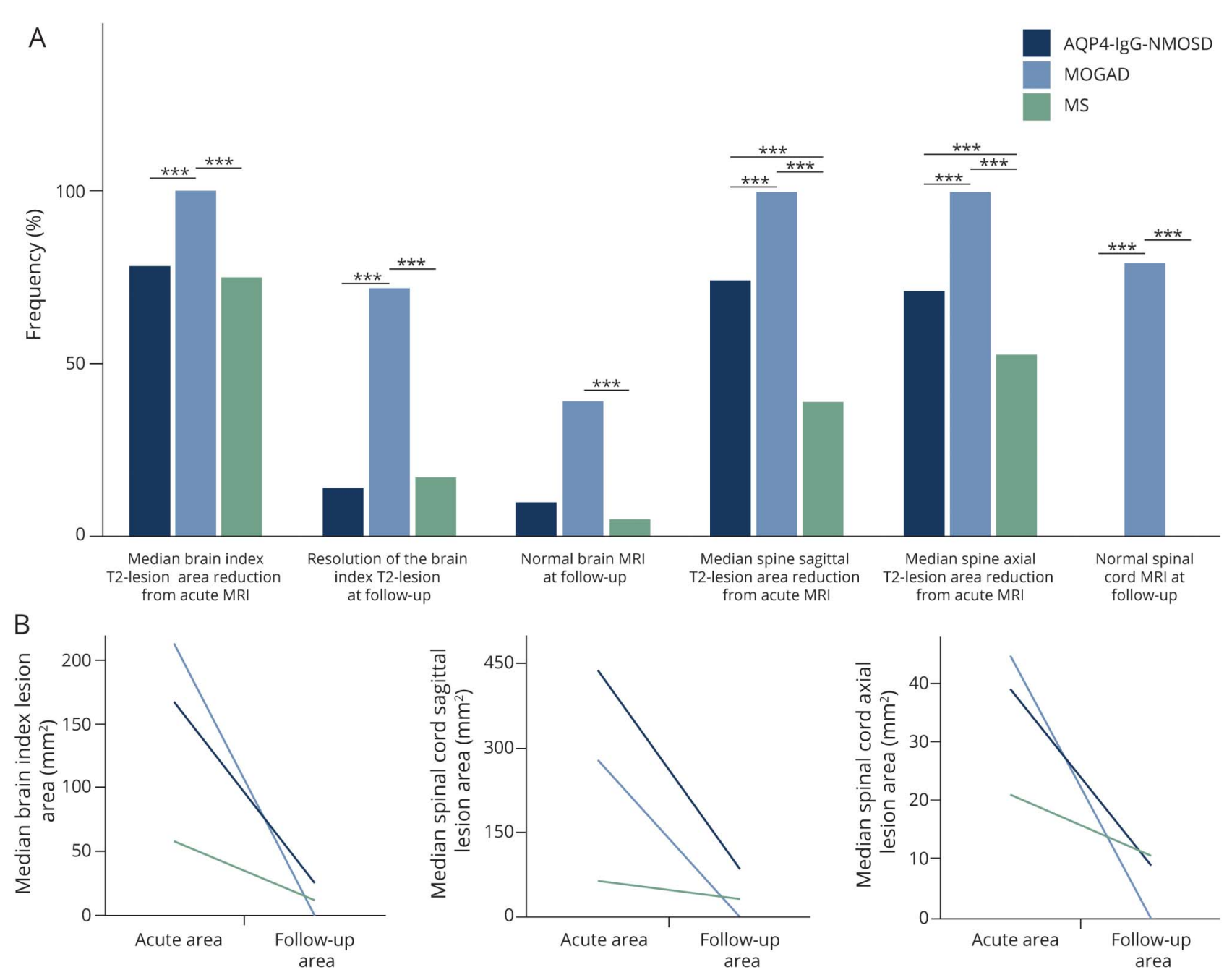

(A) Bar graphs show the different frequency of the analyzed measures in patients with aquaporin 4-immunoglobulin G-positive neuromyelitis optica spectrum disorder (AQP4-IgG-NMOSD) (dark blue bars), myelin oligodendrocyte glycoprotein-immunoglobulin G-associated disorder (MOGAD) (light blue bars), and multiple sclerosis (MS) (green bars) in the brain and spinal cord. Although the largest reduction in size was observed in AQP4-IgG-NMOSD and MOGAD associated lesions, only the latter show complete resolution of MRI abnormalities at follow-up in the majority of cases. On the contrary, MS lesions showed the smallest reduction in size at follow-up and, similarly to AQP4-IgG-NMOSD lesions, were unlikely to resolve completely. Statistically significant differences between 2 groups are indicated by the asterisks on top of the bars (***). (B) The median largest area for brain and spinal cord lesions axially, and sagittally for the spinal cord only, acutely and at follow-up MRI for the 3 groups. The exact $p$ values for different comparisons are shown in tables 2 and 3.

line with our findings and suggest MRI normalization in MOGAD may occur even after multiple attacks. A prior study specifically analyzed brain lesion evolution on MRI in 63 patients with NMOSD (95\% with AQP4-IgG), for a total of 211 acute brain lesions, and found complete resolution in $24 \%$ of these. ${ }^{17}$ The lower frequency of lesion resolution in our study could be related to the greater predominance of infratentorial lesions in our study as differences in lesion location can affect the degree of tissue injury but differences in inclusion criteria, MRI scanners, or the role of chance are other possible explanations.

Our findings have relevance for current clinical practice for helping select individuals at a higher risk of MOGAD. Resolution of most or all lesions and marked reduction in lesion size in a patient with CNS demyelinating disease and prior attacks will help select those at higher risk for MOGAD and may avoid testing in low probability situations in which the risk of false positivity increases. Given that MOG-IgG has only become available recently, there are large numbers of patients with MOGAD with serial imaging available who may have not been tested yet for MOG-IgG and for whom our findings are directly applicable. Similarly, complete or near complete T2 lesion resolution can be used, in addition to other measures, when a positive MOG-IgG test is encountered to help distinguish true vs false-positive results, particularly when antibody titer is low and the risk of false positivity is higher (up to a quarter of positive results may be false-positives when the test is performed indiscriminately in a real-life clinical setting). ${ }^{32}$

Awareness of the variability in CNS demyelinating lesion evolution across these disorders over time is important for 
optimal selection of outcome measures in clinical trials. Development of new or enlarging T2 lesions on brain MRI is often used as a primary outcome measure in MS as it allows assessment of interattack disease activity, but relies on the tendency of MS lesions to persist over time. A similar approach for MOGAD would be more problematic given the high frequency of lesion resolution as one or more transient T2 lesions could appear and disappear between interval MRI scans. This could also contribute to the low incidence of new asymptomatic $\mathrm{T} 2$ lesions reported in children with MOGAD in a prior study. ${ }^{33}$ Notably, lesion resolution in this study was not associated with less disability compared to patients with MOGAD in whom lesions persisted, suggesting that $\mathrm{T} 2$ abnormalities alone do not properly reflect functional impairment in MOGAD. ${ }^{34} \mathrm{~A}$ similar clinical-MRI discrepancy in MOGAD is sometimes seen during severe attacks accompanied by normal MRI. ${ }^{35}$ Finally, no patients had persistent gadolinium enhancement of lesions after 6 months and its presence should be a red flag for alternative nondemyelinating etiologies as illustrated by a prior study that showed it favored sarcoid myelopathy over AQP4-IgGNMOSD myelitis. ${ }^{36}$

The mechanisms that underlie the tendency of MOGAD lesions to resolve completely are not fully understood. The timing of lesion resolution in patients with MOGAD varied from days to many months and the lack of predefined intervals of MRI scans limits its assessment as it is difficult to determine at what time point between 2 MRIs the lesions may have resolved. The frequency of a normal MRI without residual T2 lesions was greater in the spine than brain and likely reflects the much higher number of brain than spine lesions, with each attack allowing greater number of opportunities to leave a residual scar (e.g., Figure 2D). It is also notable that lesion resolution was similar before and after 18 years of age. Together, these observations suggest that lesion resolution is not just a time or age-dependent process but other lesion- or patient-specific factors are likely involved. Demyelinating lesions in AQP4-IgG-NMOSD and MOGAD showed a similarly large reduction in size, some of which may reflect greater reduction in edema from large edematous T2 lesions (particularly in the spinal cord) when compared to MS. However, lesion resolution was common only with MOGAD. Greater remyelination capacity, less neuronal loss, or a predominant functional vs structural damage in MOGAD compared to AQP4-IgG-NMOSD and MS might account for this difference. Occasionally, severe edema with AQP4-IgG-NMOSD myelitis lesions in a confined spinal compartment could lead to secondary ischemia and play a role in lesion persistence, although lesion persistence was similarly observed in brain lesions associated with AQP4-IgG and other factors such as complement mediated tissue destruction are likely to contribute to residual scarring. ${ }^{37}$ This is also in line with the rarer occurrence of residual brain parenchymal T1 hypointensity after MOGAD, which supports better tissue healing and potentially less destructive lesions leading to more favorable outcomes in MOGAD. Obtaining further insight on lesion evolution from pathologic studies is limited as biopsy/autopsy assessments are generally from acute severe attacks with only scarce available data on the pathologic characteristics of lesions between attacks. ${ }^{38-40}$

This study is limited by its retrospective nature and lack of standardized MRI assessment of CNS lesions at a regular time interval on the same MRI scanner. Such studies are needed, but they may be difficult to perform. Acquiring attack MRIs, which may be undertaken at various locations (e.g., in the hospital setting), and obtaining research MRIs in the acute setting can be complicated with ethical issues if general anesthesia is required (e.g., encephalopathic patients). Moreover, given the rarity of MOGAD and AQP4-IgG-NMOSD and requirement of no interval attacks involving that same region, prospective MRI studies with sufficient power will be costly, likely require multiple centers, and could take a long time, as illustrated by this retrospective single-center study encompassing $>15$ years. Thus, this study provides valuable data while such prospective studies are awaited. The use of advanced MRI sequences or higher magnetic field scans could improve the detection of residual abnormalities not appreciable on conventional sequences but a benefit of our study with conventional MRI is the direct reproducibility to current clinical practice. Some technical aspects might bias lesion resolution assessment (e.g., we compared acute and follow-up images on different MRI scans and the variation in axial/sagittal cuts may miss small residual lesions), but the magnitude of the differences we found among the 3 groups could not be explained by these technical issues (Figures 1-4). Moreover, although comparing different MRI examinations over time introduces variability in imaging equipment, software, patient positioning, series techniques, and slice selection, these would be expected to be random variables without systematic bias. Patients received many different treatments after the initial attack and had variable follow-up durations that might have contributed to the observed differences in the 3 groups. However, both acute and maintenance therapy were very similar in patients with AQP4-IgG-NMOSD and MOGAD (Table 1), plus patients with MOGAD had shorter time interval between acute and follow-up MRI (Tables 2 and 3), and thus timing did not explain their higher frequency of lesion resolution. The proportion of patients with MS treated with anti-CD20 drugs was relatively low in our study but these drugs might increase the frequency of MS lesion resolution. Future studies will better clarify the specific effect of different acute and maintenance immunosuppressive agents (e.g., anti-CD20 vs oral immunosuppressants [azathioprine or mycophenolate mofetil] vs MS disease-modifying agents) on MRI lesion evolution. Chronic T2 lesions preceding the initial acute MRI could influence lesion resolution but our study design requiring the first ever clinical attack attributable to the region of interest reduced the risk of inclusion of such lesions. Age may affect the capacity for lesion resolution but this did not differ between children and adults in this study, although further studies with larger numbers of pediatric patients are needed. Lastly, evolution of optic nerve lesions on MRI was not analyzed in this study due to the limited availability of dedicated orbital sequences and technical issues can limit the reliability of $\mathrm{T} 2$ lesion detection in the optic nerves. 
This comparative study on the temporal evolution of MRI lesions associated with MOGAD, AQP4-IgG-NMOSD, and MS has relevance for clinical practice and our understanding of the pathogenesis of these disorders.

\section{Acknowledgment}

This study was supported by the Gianesini Research Grant from UniCredit Foundation and University of Verona, the Mayo Clinic Center for Multiple Sclerosis and Autoimmune Neurology, and the NIH National Institute of Neurological Disorders and Stroke (R01NS113828).

\section{Study Funding}

NIH National Institute of Neurologic Disorders and Stroke (R01NS113828).

\section{Disclosure}

E. Sechi, K.N. Krecke, S. Messina, and M. Buciuc report no disclosures relevant to the manuscript. S.J. Pittock reports grants, personal fees, and nonfinancial support from Alexion Pharmaceuticals, Inc., Grifols, Autoimmune Encephalitis Alliance, and MedImmune, Inc; has patent \#9,891,219 (application \#12-573942), "Methods for treating neuromyelitis optica (NMO) by administration of eculizumab to an individual that is aquaporin-4 (AQP4)IgG autoantibody positive"; and has patents pending for IgGs as biomarkers of autoimmune neurologic disorders (septin-5, Kelch-like protein 11, GFAP, PDE10A, and MAP1B). J.J. Chen reports no disclosures relevant to the manuscript. B.G. Weinshenker reports royalties from RSR Ltd., Oxford University, Hospices Civil de Lyon, and MVZ Labor PD Dr. Volkmann und Kollegen GbR for a patent of NMO-IgG as a diagnostic test for NMO and related disorders; serves as a member of an adjudication committee for clinical trials in NMO being conducted by VielaBio and Alexion pharmaceutical companies; and is a consultant for Chugai Pharma and Mitsubishi Tanabe regarding potential clinical trials for NMO. A.S. Lopez-Chiriboga reports no disclosures relevant to the manuscript. C.F. Lucchinetti received grants from Biogen, National Multiple Sclerosis Society, Kingsland Foundation, and National Institute for Neurologic Disorders and Stroke during the conduct of the study. N.L. Zalewski, J.M. Tillema, A. Kunchok, S. Monaco, P.P. Morris, J.P. Fryer, A. Nguyen, T. Greenwood, and S.B. Syc-Mazurek report no disclosures relevant to the manuscript. B.M. Keegan reports personal funding by Biogen; publishing royalties for Common Pitfalls in Multiple Sclerosis and CNS Demyelinating Diseases; and is an editorial board member of Multiple Sclerosis and Related Disorders. E.P. Flanagan reports that he has served on advisory boards for Alexion, Genentech, and Horizon Therapeutics. He has received speaker honoraria from Pharmacy Times. He received royalties from UpToDate. He was a site primary investigator in a randomized clinical trial on Inebilizumab in neuromyelitis optica spectrum disorder run by Medimmune/Viela-Bio/Horizon Therapeutics. He has received funding from the NIH
(R01NS113828). He is a member of the medical advisory board of the MOG project. He is an editorial board member of the Journal of the Neurological Sciences and Neuroimmunology Reports.

\section{Publication History}

Received by Neurology December 30, 2020. Accepted in final form June 11, 2021.

Appendix Authors

\begin{tabular}{|c|c|c|}
\hline Name & Location & Contribution \\
\hline Elia Sechi, MD & $\begin{array}{l}\text { Mayo Clinic, } \\
\text { Rochester, } \\
\text { MN; University } \\
\text { of Verona, Italy }\end{array}$ & $\begin{array}{l}\text { Designed and conceptualized the } \\
\text { study; drafted the manuscript and } \\
\text { figures; analyzed and interpreted the } \\
\text { data }\end{array}$ \\
\hline $\begin{array}{l}\text { Karl N. Krecke, } \\
\text { MD }\end{array}$ & $\begin{array}{l}\text { Mayo Clinic, } \\
\text { Rochester, MN }\end{array}$ & $\begin{array}{l}\text { Interpreted the data; revised the } \\
\text { manuscript for intellectual content }\end{array}$ \\
\hline $\begin{array}{l}\text { Steven A. } \\
\text { Messina, MD }\end{array}$ & $\begin{array}{l}\text { Mayo Clinic, } \\
\text { Rochester, MN }\end{array}$ & $\begin{array}{l}\text { Interpreted the data; revised the } \\
\text { manuscript for intellectual content }\end{array}$ \\
\hline $\begin{array}{l}\text { Marina Buciuc, } \\
\text { MD }\end{array}$ & $\begin{array}{l}\text { Mayo Clinic, } \\
\text { Rochester, MN }\end{array}$ & $\begin{array}{l}\text { Interpreted the data; revised the } \\
\text { manuscript for intellectual content }\end{array}$ \\
\hline $\begin{array}{l}\text { Sean J. Pittock, } \\
\text { MD }\end{array}$ & $\begin{array}{l}\text { Mayo Clinic, } \\
\text { Rochester, MN }\end{array}$ & $\begin{array}{l}\text { Interpreted the data; revised the } \\
\text { manuscript for intellectual content }\end{array}$ \\
\hline $\begin{array}{l}\text { John J. Chen, MD, } \\
\text { PhD }\end{array}$ & $\begin{array}{l}\text { Mayo Clinic, } \\
\text { Rochester, MN }\end{array}$ & $\begin{array}{l}\text { Interpreted the data; revised the } \\
\text { manuscript for intellectual content }\end{array}$ \\
\hline $\begin{array}{l}\text { Brian G. } \\
\text { Weinshenker, MD }\end{array}$ & $\begin{array}{l}\text { Mayo Clinic, } \\
\text { Rochester, MN }\end{array}$ & $\begin{array}{l}\text { Interpreted the data; revised the } \\
\text { manuscript for intellectual content }\end{array}$ \\
\hline $\begin{array}{l}\text { A. Sebastian } \\
\text { Lopez-Chiriboga, } \\
\text { MD }\end{array}$ & $\begin{array}{l}\text { Mayo Clinic, } \\
\text { Jacksonville, FL }\end{array}$ & $\begin{array}{l}\text { Interpreted the data; revised the } \\
\text { manuscript for intellectual content }\end{array}$ \\
\hline $\begin{array}{l}\text { Claudia F. } \\
\text { Lucchinetti, MD }\end{array}$ & $\begin{array}{l}\text { Mayo Clinic, } \\
\text { Rochester, MN }\end{array}$ & $\begin{array}{l}\text { Interpreted the data; revised the } \\
\text { manuscript for intellectual content }\end{array}$ \\
\hline $\begin{array}{l}\text { Nicholas L. } \\
\text { Zalewski, MD }\end{array}$ & $\begin{array}{l}\text { Mayo Clinic, } \\
\text { Rochester, MN }\end{array}$ & $\begin{array}{l}\text { Interpreted the data; revised the } \\
\text { manuscript for intellectual content }\end{array}$ \\
\hline $\begin{array}{l}\text { Jan Mendelt } \\
\text { Tillema, MD }\end{array}$ & $\begin{array}{l}\text { Mayo Clinic, } \\
\text { Scottsdale, AZ }\end{array}$ & $\begin{array}{l}\text { Interpreted the data; revised the } \\
\text { manuscript for intellectual content }\end{array}$ \\
\hline $\begin{array}{l}\text { Amy Kunchok, } \\
\text { MD }\end{array}$ & $\begin{array}{l}\text { Mayo Clinic, } \\
\text { Rochester, MN }\end{array}$ & $\begin{array}{l}\text { Interpreted the data; revised the } \\
\text { manuscript for intellectual content }\end{array}$ \\
\hline $\begin{array}{l}\text { Salvatore } \\
\text { Monaco, MD }\end{array}$ & $\begin{array}{l}\text { University of } \\
\text { Verona, Italy }\end{array}$ & $\begin{array}{l}\text { Interpreted the data; revised the } \\
\text { manuscript for intellectual content }\end{array}$ \\
\hline $\begin{array}{l}\text { Padraig P. Morris, } \\
\text { MD }\end{array}$ & $\begin{array}{l}\text { Mayo Clinic, } \\
\text { Rochester, MN }\end{array}$ & $\begin{array}{l}\text { Interpreted the data; revised the } \\
\text { manuscript for intellectual content }\end{array}$ \\
\hline $\begin{array}{l}\text { James P. Fryer, } \\
\text { MS }\end{array}$ & $\begin{array}{l}\text { Mayo Clinic, } \\
\text { Rochester, MN }\end{array}$ & $\begin{array}{l}\text { Interpreted the data; revised the } \\
\text { manuscript for intellectual content }\end{array}$ \\
\hline Adam Nguyen & $\begin{array}{l}\text { Mayo Clinic, } \\
\text { Rochester, MN }\end{array}$ & $\begin{array}{l}\text { Interpreted the data; revised the } \\
\text { manuscript for intellectual content }\end{array}$ \\
\hline $\begin{array}{l}\text { Tammy } \\
\text { Greenwood, MS }\end{array}$ & $\begin{array}{l}\text { Mayo Clinic, } \\
\text { Rochester, MN }\end{array}$ & $\begin{array}{l}\text { Interpreted the data; revised the } \\
\text { manuscript for intellectual content }\end{array}$ \\
\hline $\begin{array}{l}\text { Stephanie B. Syc- } \\
\text { Mazurek, MD, } \\
\text { PhD }\end{array}$ & $\begin{array}{l}\text { Mayo Clinic, } \\
\text { Rochester, MN }\end{array}$ & $\begin{array}{l}\text { Interpreted the data; revised the } \\
\text { manuscript for intellectual content }\end{array}$ \\
\hline $\begin{array}{l}\text { B. Mark Keegan, } \\
\text { MD }\end{array}$ & $\begin{array}{l}\text { Mayo Clinic, } \\
\text { Rochester, MN }\end{array}$ & $\begin{array}{l}\text { Interpreted the data; revised the } \\
\text { manuscript for intellectual content }\end{array}$ \\
\hline $\begin{array}{l}\text { Eoin P. Flanagan, } \\
\text { MD }\end{array}$ & $\begin{array}{l}\text { Mayo Clinic, } \\
\text { Rochester, MN }\end{array}$ & $\begin{array}{l}\text { Designed and conceptualized the } \\
\text { study; analyzed and interpreted the } \\
\text { data; study supervision }\end{array}$ \\
\hline
\end{tabular}




\section{References}

1. Flanagan EP. Neuromyelitis optica spectrum disorder and other non-multiple sclerosis central nervous system inflammatory diseases. Continuum. 2019;25(3):815-844

2. Lennon VA, Wingerchuk DM, Kryzer TJ, et al. A serum autoantibody marker of neuromyelitis optica: distinction from multiple sclerosis. Lancet. 2004;364(9451):2106-2112.

3. O'Connor KC, McLaughlin KA, De Jager PL, et al.. Self-antigen tetramers discriminate between myelin autoantibodies to native or denatured protein. Nat Med. 2007; 13(2):211-217.

4. Reindl M, Waters P. Myelin oligodendrocyte glycoprotein antibodies in neurological disease. Nat Rev Neurol. 2019;15(2):89-102.

5. Dubey D, Pittock SJ, Krecke KN, et al. Clinical, radiologic, and prognostic features of myelitis associated with myelin oligodendrocyte glycoprotein autoantibody. JAMA Neurol. 2018;76(3):301-309.

6. Jurynczyk M, Geraldes R, Probert F, et al. Distinct brain imaging characteristics of autoantibody-mediated CNS conditions and multiple sclerosis. Brain. 2017;140(3): 617-627.

7. Mariano R, Messina S, Kumar K, Kuker W, Leite MI, Palace J. Comparison of clinical outcomes of transverse myelitis among adults with myelin oligodendrocyte glycoprotein antibody vs aquaporin-4 antibody disease. JAMA Netw Open. 2019;2(10):e1912732.

8. Asnafi S, Morris PP, Sechi E, et al. The frequency of longitudinally extensive transverse myelitis in MS: a population-based study. Mult Scler Relat Disord. 2020;37:101487.

9. Cobo-Calvo A, Ruiz A, Maillart E, et al. Clinical spectrum and prognostic value of CNS MOG autoimmunity in adults: the MOGADOR study. Neurology. 2018;90(21): e1858-e1869.

10. Kim HJ, Paul F, Lana-Peixoto MA, et al. MRI characteristics of neuromyelitis optica spectrum disorder: an international update. Neurology. 2015;84(11):1165-1173.

11. Ramanathan S, Mohammad S, Tantsis E, et al. Clinical course, therapeutic responses and outcomes in relapsing MOG antibody-associated demyelination. J Neurol Neurosurg Psychiatry. 2018;89(2):127-137.

12. Lopez-Chiriboga S, Sechi E, Buciuc M, et al. Long-term outcomes in patients with myelin oligodendrocyte glycoprotein immunoglobulin G-associated disorder. JAMA Neurol. 2020;77(12):1575-1577.

13. Wingerchuk DM, Pittock SJ, Lucchinetti CF, Lennon VA, Weinshenker BG. A secondary progressive clinical course is uncommon in neuromyelitis optica. Neurology. 2007;68(8):603-605.

14. Paolilo RB, Hacohen Y, Yazbeck E, et al. Treatment and outcome of aquaporin-4 antibody-positive NMOSD: a multinational pediatric study. Neurol Neuroimmunol Neuroinflamm. 2020;7(5):e837.

15. Filippi M, Preziosa P, Banwell BL, et al. Assessment of lesions on magnetic resonance imaging in multiple sclerosis: practical guidelines. Brain. 2019;142(7):1858-1875.

16. Kitley J, Waters $\mathrm{P}$, Woodhall $\mathrm{M}$, et al. Neuromyelitis optica spectrum disorders with aquaporin-4 and myelin-oligodendrocyte glycoprotein antibodies: a comparative study. JAMA Neurol. 2014;71(3):276-283.

17. Kim SH, Huh SY, Hyun JW, et al. A longitudinal brain magnetic resonance imaging study of neuromyelitis optica spectrum disorder. PLoS One. 2014;9(9):e108320.

18. Jarius S, Paul F, Aktas O, et al. MOG encephalomyelitis: international recommendations on diagnosis and antibody testing. J Neuroinflamm. 2018;15(1):134.

19. Thompson AJ, Banwell BL, Barkhof F, et al. Diagnosis of multiple sclerosis: 2017 revisions of the McDonald criteria. Lancet Neurol. 2018;17(2):162-173.

20. Wingerchuk DM, Banwell B, Bennett JL, et al. International consensus diagnostic criteria for neuromyelitis optica spectrum disorders. Neurology. 2015;85(2): 177-189.
21. Banks SA, Morris PP, Chen JJ, et al. Brainstem and cerebellar involvement in MOGIgG-associated disorder versus aquaporin-4-IgG and MS. J Neurol Neurosurg Psychiatry. Epub 2020 Dec 28.

22. Waters PJ, McKeon A, Leite MI, et al. Serologic diagnosis of NMO: a multicenter comparison of aquaporin-4-IgG assays. Neurology. 2012;78(9):665-671; discussion 669.

23. Mariano R, Messina S, Roca-Fernandez A, Leite MI, Kong Y, Palace JA. Quantitative spinal cord MRI in MOG-antibody disease, neuromyelitis optica and multiple sclerosis. Brain. 2021;144(1):198-212.

24. Wingerchuk DM, Hogancamp WF, O’Brien PC, Weinshenker BG. The clinical course of neuromyelitis optica (Devic's syndrome). Neurology. 1999;53(5):1107-1114.

25. Absinta M, Sati P, Masuzzo F, et al. Association of chronic active multiple sclerosis lesions with disability in vivo. JAMA Neurol. 2019;76(12):1474-1483.

26. Lucchinetti CF, Bruck W, Rodriguez M, Lassmann H. Distinct patterns of multiple sclerosis pathology indicates heterogeneity on pathogenesis. Brain Pathol. 1996;6(7): 259-274.

27. Sechi E, Keegan BM, Kaufmann TJ, Kantarci OH, Weinshenker BG, Flanagan EP. Unilateral motor progression in MS: association with a critical corticospinal tract lesion. Neurology. 2019;93(7):e628-e634.

28. Sechi E, Messina S, Keegan BM, et al. Critical spinal cord lesions associate with secondary progressive motor impairment in long-standing MS: a population-based case-control study. Mult Scler. 2021;27(5):667-673.

29. Keegan BM, Kaufmann TJ, Weinshenker BG, et al. Progressive solitary sclerosis gradual motor impairment from a single CNS demyelinating lesion. Neurology. 2016, 87(16):1713-1719.

30. Lechner C, Baumann M, Hennes EM, et al. Antibodies to MOG and AQP4 in children with neuromyelitis optica and limited forms of the disease. J Neurol Neurosurg Psychiatry. 2016;87(8):897-905.

31. Waters P, Fadda G, Woodhall M, et al. Serial anti-myelin oligodendrocyte glycoprotein antibody analyses and outcomes in children with demyelinating syndromes. JAMA Neurol. 2019;77(1):82-93.

32. Sechi EB M, Pittock SJ, Chen JJ, et al. Positive predictive value of myelin oligodendrocyte glycoprotein autoantibody testing. JAMA Neurol. 2021;78(6):741-746.

33. Fadda G, Banwell B, Waters $P$, et al. Silent new brain MRI lesions in children with MOG-antibody associated disease. Ann Neurol. 2020;89(2):408-413.

34. Buciuc M, Sechi E, Flanagan EP, Lopez-Chiriboga AS. Unfavorable outcome in highly relapsing MOGAD encephalitis. J Neurol Sci. 2020;418:117088.

35. Sechi E, Krecke KN, Pittock SJ, et al. Frequency and characteristics of MRI-negative myelitis associated with MOG autoantibodies. Mult Scler. 2021;27(2):303-308.

36. Flanagan EP, Kaufmann TJ, Krecke KN, et al. Discriminating long myelitis of neuromyelitis optica from sarcoidosis. Ann Neurol. 2016;79(3):437-447.

37. Hinson SR, Romero MF, Popescu BF, et al. Molecular outcomes of neuromyelitis optica (NMO)-IgG binding to aquaporin-4 in astrocytes. Proc Natl Acad Sci USA 2012;109(4):1245-1250.

38. Hoftberger R, Guo Y, Flanagan EP, et al. The pathology of central nervous system inflammatory demyelinating disease accompanying myelin oligodendrocyte glycoprotein autoantibody. Acta Neuropathol. 2020;139(5):875-892.

39. Lucchinetti CF, Guo Y, Popescu BF, Fujihara K, Itoyama Y, Misu T. The pathology of an autoimmune astrocytopathy: lessons learned from neuromyelitis optica. Brain Pathol. 2014;24(1):83-97.

40. Takai Y, Misu T, Kaneko K, et al. Myelin oligodendrocyte glycoprotein antibodyassociated disease: an immunopathological study. Brain. 2020;143(5):1431-1446. 


\section{Neurology}

\section{Comparison of MRI Lesion Evolution in Different Central Nervous System Demyelinating Disorders}

Elia Sechi, Karl N. Krecke, Steven A. Messina, et al. Neurology 2021;97;e1097-e1109 Published Online before print July 14, 2021

DOI 10.1212/WNL.0000000000012467

\section{This information is current as of July 14, 2021}

\section{Updated Information \& Services}

\section{References}

Citations

Permissions \& Licensing

Reprints including high resolution figures, can be found at: http://n.neurology.org/content/97/11/e1097.full

This article cites 39 articles, 11 of which you can access for free at: http://n.neurology.org/content/97/11/e1097.full\#ref-list-1

This article has been cited by 4 HighWire-hosted articles: http://n.neurology.org/content/97/11/e1097.full\#\#otherarticles

Information about reproducing this article in parts (figures,tables) or in its entirety can be found online at:

http://www.neurology.org/about/about_the_journal\#permissions

Information about ordering reprints can be found online:

http://n.neurology.org/subscribers/advertise

Neurology ${ }^{\circledR}$ is the official journal of the American Academy of Neurology. Published continuously since 1951, it is now a weekly with 48 issues per year. Copyright Copyright ( 2021 The Author(s). Published by Wolters Kluwer Health, Inc. on behalf of the American Academy of Neurology.. All rights reserved. Print ISSN: 0028-3878. Online ISSN: 1526-632X.

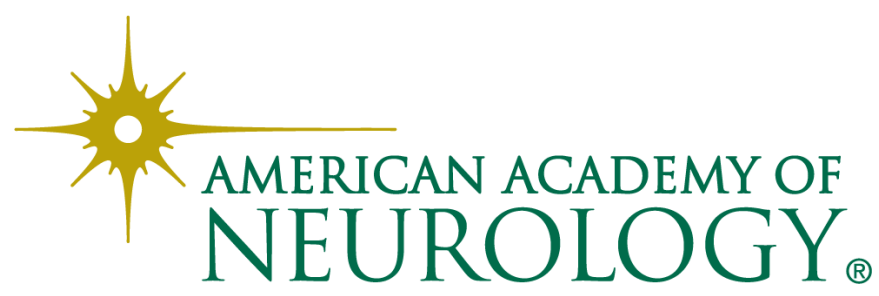

